\title{
DETERMINAÇÃo VOLTAMÉTRICA POR REDISSOLUÇÃo ANÓDICA DE Cu(II) EM ÁGUAS RESIDUÁRIAS EMPREGANDO UM ELETRODO DE PASTA DE CARBONO MODIFICADO COM QUITOSANA
}

Bruno Campos Janegitz, Luiz Humberto Marcolino Junior e Orlando Fatibello-Filho*

Departamento de Química, Universidade Federal de São Carlos, CP 676, 13560-970 São Carlos - SP, Brasil

Recebido em 24/10/06; aceito em 9/3/07; publicado na web em 6/8/07

\begin{abstract}
ANODIC STRIPPING VOLTAMMETRIC DETERMINATION OF COPPER (II) IN WASTEWATERS USING A CARBON PASTE ELECTRODE MODIFIED WITH CHITOSAN. The electrochemical applications of a CPE modified with chitosan for the determination of $\mathrm{Cu}$ (II) in wastewater samples using anodic stripping voltammetry are described. The best voltammetric response was observed for a paste containing $25 \% \mathrm{~m} / \mathrm{m}$ of chitosan. A $0.10 \mathrm{~mol} \mathrm{~L}^{-1} \mathrm{NaNO}_{3}$ solution (pH 6.5) as supporting electrolyte, a pre-concentration potential of $-0.20 \mathrm{~V}$, pre-concentration time of $270 \mathrm{~s}$ and a scan rate of $25 \mathrm{mV} \mathrm{s}^{-1}$ were selected. The calibration graph was linear in the $\mathrm{Cu}(\mathrm{II})$ concentration range from $2.0 \times 10^{-7}$ to $7.4 \times 10^{-6} \mathrm{~mol} \mathrm{~L}^{-1}$, with a detection limit of $8.3 \times 10^{-8} \mathrm{~mol} \mathrm{~L}^{-1}$.
\end{abstract}

Keywords: chitosan; copper(II); stripping analysis.

\section{INTRODUÇÃO}

O desenvolvimento de procedimentos analíticos que permitam determinar concentrações baixas de íons metálicos em águas naturais e/ou residuárias é de extremo interesse e vem sendo objeto de estudo recente em química analítica. Com o advento das técnicas eletroanalíticas, os sensores eletroquímicos ganharam um importante espaço nas diversas áreas de pesquisa ${ }^{1,2}$.

O desenvolvimento e aplicação de eletrodos modificados $(\mathrm{EM})^{3-7}$, tem tido crescente interesse nas áreas de ciências e tecnologia, com aplicação em diversos campos de pesquisa, tais como eletrocatálise, síntese eletroorgânica, conversão de energia solar e, principalmente, em eletroanálise e sensores químicos. A denominação de eletrodos quimicamente modificados foi sugerida por Murray et al. na década de 70 para definir eletrodos com espécies quimicamente ativas devidamente imobilizadas na superfície desses dispositivos ${ }^{8}$. O EM é construído adicionando-se espécies quimicamente ativas imobilizadas no substrato do eletrodo, com o objetivo de pré-estabelecer e controlar a natureza físico-química da interface eletrodo/solução. A modificação do eletrodo normalmente confere a este as características físico-químicas (reatividade, seletividade etc.) do modificador, possibilitando assim o desenvolvimento de dispositivos com resposta adequada para vários propósitos e aplicações. Nesse aspecto, são encontradas na literatura diversas revisões que descrevem as diferentes estratégias para construção de eletrodos modificados ${ }^{3-9}$.

A introdução da pasta de carbono e suas primeiras aplicações estão relacionadas com o grupo de Adams ${ }^{10}$ que estudou algumas características básicas e algumas aplicações para os eletrodos de pasta de carbono. No final da década de 70 surgiram vários trabalhos empregando modificações dos eletrodos, sendo o início dos anos 80 um marco na história dos eletrodos de pasta de carbono modificados, quando se desenvolveu um sensor modificado com dimetilglioxima para a determinação de íons $\mathrm{Ni}(\mathrm{II})^{11}$. Neste trabalho, verificou-se que o uso de um reagente seletivo clássico proporcionou aumento significativo da seletividade do eletrodo.

A superfície dos eletrodos de pasta de carbono é muito complexa, com muitas possibilidades de interações. O líquido orgânico aglutinante serve para fixar a pasta ao eletrodo, preencher as

*e-mail: bello@dq.ufscar.br cavidades entre as partículas de grafite e "isolar" o mesmo do contato com soluções aquosas; também deve ser eletroinativo, possuir baixa volatilidade e não conter impurezas ${ }^{12}$.

A quitosana ${ }^{13,14}$ (Figura 1) é um biopolímero, obtida a partir da reação de desacetilação da quitina (presente nas cascas de crustáceos e fungos). Esse biopolímero vem sendo aplicado na remoção de metais pesados em águas residuárias ${ }^{15}$, como também na determinação destes metais em soluções aquosas. Revisões das inúmeras possibilidades de aplicações da quitina e da quitosana foram apresentadas recentemente ${ }^{16,17}$. Foi destacada a versatilidade física que pode ser obtida a partir desses polímeros, como a obtenção de fibras, filmes, géis, microesferas e membranas. Além disso, a capacidade da quitosana de formar complexos com íons metálicos tem despertado o interesse de diversos pesquisadores ${ }^{18,19}$.

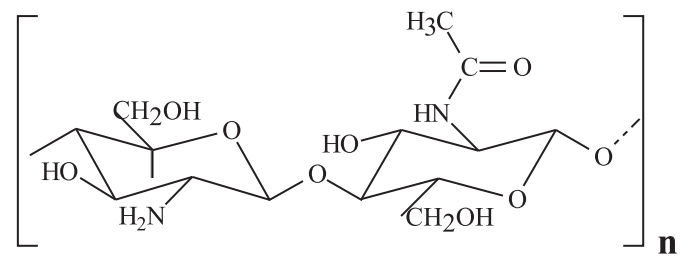

Figura 1. Estrutura da quitosana

São encontrados na literatura diversos trabalhos utilizando quitosana como agente modificador de eletrodos, geralmente associados a uma enzima, formando um biossensor ${ }^{20-25}$. Tan e colaboradores $^{26}$ utilizaram a quitosana como material para a formação de um filme híbrido com sílica, sendo usado posteriormente para a imobilização da enzima colesterol oxidase em um eletrodo de carbono vítreo modificado com Azul da Prússia. O sensor foi aplicado para a determinação de colesterol em amostras de sangue em um intervalo de concentração de 4,0 x 10-6 a 7,0 x $10^{-4} \mathrm{~mol} \mathrm{~L}^{-1}$, com um limite de detecção de $1,0 \times 10^{-6} \mathrm{~mol} \mathrm{~L}^{-1}$. Em um outro trabalho, de Oliveira et al. ${ }^{27}$ imobilizaram um extrato bruto de giló (Solanum gilo) como fonte de peroxidase em uma matriz de quitosana e depois construíram um biossensor de pasta de carbono para a determinação de rutina em formulações farmacêuticas. O biossensor exibiu uma resposta linear no intervalo de concentração de rutina de $3,4 \times 10^{-7}$ a 7,2 x $10^{-6} \mathrm{~mol} \mathrm{~L}^{-1}$, com um limite de detecção de $2,0 \times 10^{-8} \mathrm{~mol} \mathrm{~L}^{-1}$. 
No presente trabalho descreve-se o desenvolvimento de um eletrodo de pasta de carbono modificado com quitosana para a determinação de íons cobre (II), utilizando-se a técnica de voltametria de redissolução anódica. O emprego da quitosana como agente modificador para sensores de íons metálicos tem sido pouco explorado, apesar da grande afinidade deste material por cátions metálicos.

\section{PARTE EXPERIMENTAL}

Os experimentos eletroquímicos foram realizados em uma célula de compartimento único de $15 \mathrm{~mL}$, confeccionada em vidro Pyrex. Foi utilizado um sistema de três eletrodos, sendo que o eletrodo de trabalho foi o eletrodo quimicamente modificado com quitosana (EPCM), o de referência, um eletrodo de $\mathrm{Ag} / \mathrm{AgCl}\left(\mathrm{KCl} 3 \mathrm{~mol} \mathrm{~L}{ }^{-1}\right) \mathrm{e}$ o auxiliar, um eletrodo de platina. As medidas eletroquímicas foram executadas com um potenciostato/galvanostato Autolab PGSTAT12 (Eco Chemie) gerenciado pelo software GPES 4.9. As medidas de $\mathrm{pH}$ das soluções foram realizadas com um pHmetro digital Orion, modelo EA 940. Para as medidas espectrofotométricas utilizou-se um aparelho de absorção atômica (Varian modelo Gemini).

Foram homogeneizados $600 \mathrm{mg}$ de pó de grafite (Aldrich) $(60 \%$ $\mathrm{m} / \mathrm{m}$ ) junto com $250 \mathrm{mg}$ de quitosana (Aldrich) $(25 \% \mathrm{~m} / \mathrm{m})$ e 150 mg de óleo mineral (Aldrich) $(15 \% \mathrm{~m} / \mathrm{m})$ em um béquer contendo aproximadamente $10 \mathrm{~mL}$ de hexano (Synth). Deixou-se a mistura sob agitação mecânica até a total evaporação do solvente. A pasta obtida foi inserida em uma seringa plástica para insulina $(90 \mathrm{~mm}$ de altura e diâmetro interno da ponta de $2,6 \mathrm{~mm}$ ). O eletrodo foi inserido em uma célula eletroquímica contendo o eletrodo auxiliar (platina) e o de referência $\left(\mathrm{Ag} / \mathrm{AgCl}, \mathrm{KCl} 3 \mathrm{~mol} \mathrm{~L}^{-1}\right)$ sendo, em seguida realizadas as medidas empregando-se o potenciostato mencionado. Todos os parâmertros foram avaliados a fim de se obter o melhor desempenho do sensor na determinação de íons $\mathrm{Cu}(\mathrm{II})$.

\section{RESULTADOS E DISCUSSÃO}

Utilizando as técnicas de voltametria cíclica e linear foram obtidos alguns resultados e/ou figuras de mérito do sistema analítico, como tempo de acumulação, características químicas do eletrólito suporte nas etapas de acumulação e de redissolução, região de resposta linear, sensibilidade, limites de quantificação e detecção, reprodutibilidade etc. Efeitos de interferentes em potencial também foram avaliados.

\section{Estudo dos constituintes da pasta}

Estudou-se inicialmente a composição da pasta de carbono variando a porcentagem de pó de grafite entre 50 e $65 \% \mathrm{~m} / \mathrm{m}$, sendo a composição de $60 \% \mathrm{~m} / \mathrm{m}$ selecionada por proporcionar melhor perfil voltamétrico e maior sinal analítico. Também foi estudado o efeito da concentração do biopolímero na pasta nas porcentagens de 20 , 25 e $30 \% \mathrm{~m} / \mathrm{m}$. Observou-se maior magnitude de corrente para uma composição da pasta de carbono contendo $25 \% \mathrm{~m} / \mathrm{m}$ de quitosana, $60 \% \mathrm{~m} / \mathrm{m}$ de pó de grafite e $15 \% \mathrm{~m} / \mathrm{m}$ de óleo mineral. Para uma concentração de quitosana menor que $25 \%(\mathrm{~m} / \mathrm{m})$, o sinal analítico diminuiu consideravelmente devido à menor quantidade de quitosana disponível para a complexação dos íons metálicos contidos na solução. Na pasta contendo quitosana $30 \% \mathrm{~m} / \mathrm{m}$, a diminuição da percentagem de pó de grafite compromete a transferência eletrônica devido à baixa concentração do material condutor na pasta, diminuindo desta forma a magnitude de corrente.

\section{Estudo do eletrólito suporte}

Realizou-se o estudo do eletrólito suporte contido na célula eletroquímica para a realização das medidas voltamétricas. Foram avaliadas as seguintes soluções: $\mathrm{KCl}, \mathrm{NaCl}, \mathrm{NaNO}_{3}$ e $\mathrm{KNO}_{3}$ nas concentrações variando de 0,10 a $1,0 \mathrm{~mol} \mathrm{~L}^{-1}$, onde em solução de $\mathrm{NaNO}_{3}$ o eletrodo apresentou a melhor resposta voltamétrica para o cátion $\mathrm{Cu}$ (II). Em seguida, realizou-se o estudo do efeito da concentração da solução de nitrato de sódio em concentrações variando de 0,010 a 2,0 mol L-1, sendo a concentração de $0,10 \mathrm{~mol} \mathrm{~L}^{-1}$ selecionada, uma vez que nesta concentração obteve-se a melhor resposta analítica (maior razão sinal/ruído).

\section{Estudo do pH}

Estudou-se o comportamento voltamétrico do EPCM modificado com $25 \% \mathrm{~m} / \mathrm{m}$ de quitosana com uma solução de $\mathrm{NaNO}_{3} 0,1$ mol $\mathrm{L}^{-1}$ no intervalo de $\mathrm{pH}$ de 2,5 a 7,0.

$$
\begin{aligned}
& \mathrm{Cu}^{2+}(\mathrm{aq})+2 \mathrm{H}_{2} \mathrm{O}(\mathrm{aq}) \stackrel{\mathrm{Kh}_{1}}{=} \mathrm{CuOH}^{+}(\mathrm{aq})+\mathrm{H}_{3} \mathrm{O}^{+}(\mathrm{aq}) \\
& \mathrm{CuOH}^{+}(\mathrm{aq})+2 \mathrm{H}_{2} \mathrm{O}(\mathrm{aq}) \stackrel{\mathrm{Kh}_{2}}{=} \mathrm{Cu}(\mathrm{OH})_{2}(\mathrm{~s})+\mathrm{H}_{3} \mathrm{O}^{+}(\mathrm{aq})
\end{aligned}
$$

Os resultados apresentados mostram um aumento pronunciado do sinal analítico em $\mathrm{pH} 3,5$. Neste $\mathrm{pH}$, parte da quitosana imobilizada intumesce e começa a sofrer processo de dissolução aumentando assim a rugosidade da superfície do sensor, ocasionando maior contato dos íons $\mathrm{Cu}$ (II) com a superfície do eletrodo. Ademais, o maior sinal analítico observado pode estar associado também à distribuição das espécies de cobre em solução, em diferentes valores de pH. Em valores de pH acima de 3,5, dependendo da concentração de $\mathrm{Cu}$ (II) em solução, poderá haver hidrólise parcial ou total deste íon metálico como mostrado nas Equações 1 e 2, diminuindo a concentração de $\mathrm{Cu}$ (II) livre e, conseqüentemente, diminuindo o sinal analítico, uma vez que a interação da quitosana, imobilizada no eletrodo pela espécie $\mathrm{CuOH}^{+}$, deve ser menor que aquela entre quitosana e $\mathrm{Cu}^{2+}$ e, aquela com o $\mathrm{Cu}(\mathrm{OH})_{2}$ (s), desprezível.

Apesar da maior magnitude do sinal analítico nesta concentra-

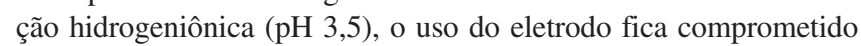
devido à rápida degradação da pasta de carbono, prejudicando a reprodutibilidade e a repetibilidade dos resultados obtidos.

Segundo dados da literatura ${ }^{28}$, a maior estabilidade entre quitosana e $\mathrm{Cu}(\mathrm{II})$ ocorre em $\mathrm{pH}$ 6,0-7,0, corroborando assim o valor de $\mathrm{pH}(6,5)$ empregado no eletrólito suporte nos estudos voltamétricos.

\section{Estudo do potencial de pré-concentração}

Estudou-se o potencial ideal para a pré-concentração de $\mathrm{Cu}(\mathrm{II})$ no EPCM com quitosana em um intervalo de potencial de $-0,3$ a $-0,1$ $\mathrm{V}$. A resposta analítica aumentou no intervalo de $-0,1$ a $-0,2 \mathrm{~V}$, mantendo-se constante em potenciais mais negativos. O potencial de $-0,2 \mathrm{~V}$ foi então selecionado.

\section{Estudo do tempo de pré-concentração}

Avaliou-se a pré-concentração de cobre no eletrodo aplicandose um potencial de $-0,2 \mathrm{~V}$ em um intervalo de 0 a $330 \mathrm{~s}$, adicionando-se diferentes alíquotas de uma solução de $\mathrm{Cu}$ (II) $1,0 \times 10^{-3} \mathrm{~mol}$ $\mathrm{L}^{-1}$. Em seguida, realizou-se a etapa de redissolução anódica utilizando voltametria linear em um intervalo de potenciais de $-0,2$ a $0,6 \mathrm{~V}$. O tempo de pré-concentração que proporcionou um melhor perfil voltamétrico, além de maior magnitude de corrente, foi o de $270 \mathrm{~s}$, sendo este tempo adotado para os estudos posteriores, como observado na Figura 2. 


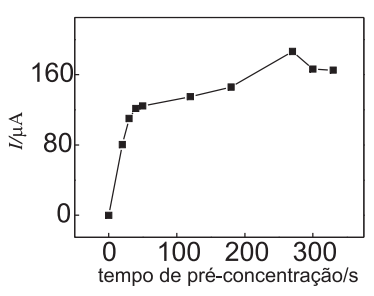

Figura 2. Efeito do tempo de pré-concentração sobre o sinal analítico para uma solução de Cu (II) $1,0 \times 10^{-3} \mathrm{~mol} \mathrm{~L}^{-1}$ em solução de $\mathrm{NaNO}_{3}$ 0,1 mol L $\mathrm{L}^{-1}$; $p H=6,5, v=25 \mathrm{mVs}^{-1}, a 25^{\circ} \mathrm{C}$

\section{Estudo da velocidade de varredura}

$\mathrm{Na}$ etapa de redissolução de $\mathrm{Cu}(0)$ do eletrodo empregando-se voltametria de redissolução anódica, estudou-se a velocidade de varredura de potencial de 5 a $50 \mathrm{mV} \mathrm{s}^{-1}$, sendo escolhida a velocidade de varredura de potencial de $25 \mathrm{mV} \mathrm{s}^{-1}$, uma vez que se obteve o melhor perfil do sinal analítico. Os dados experimentais mostraram que a relação da corrente de pico com a velocidade de varredura (Ipa vs. v) é característica de um processo adsortivo, indicando assim a complexação dos íons $\mathrm{Cu}$ (II) na superfície do eletrodo, conforme descrito por $\mathrm{Lu}$ et al. ${ }^{29}$.

\section{Estudo do comportamento voltamétrico do EPCM com quitosana}

O ciclovoltamograma obtido após um tempo de pré-concentração de $270 \mathrm{~s}$, em circuito fechado aplicando-se um potencial de $-0,2 \mathrm{~V}$ apresenta um pico anódico de oxidação de $\mathrm{Cu}(0)$ a $\mathrm{Cu}(\mathrm{II})$ em torno de $+0,4$ V. A Figura 3 apresenta os ciclovoltamogramas obtidos com o eletrodo de pasta de carbono não modificado (EPC) na ausência (A) e na presença (C) de uma solução contendo íons $\mathrm{Cu}$ (II) $1,0 \times 10^{-3} \mathrm{~mol} \mathrm{~L}^{-1} \mathrm{e}$, também, os ciclovoltamogramas obtidos com o eletrodo de pasta de carbono modificado com quitosana (EPCM-QTS) na ausência (B) e na presença (D) da mesma solução contendo íons Cu(II). Observa-se claramente um aumento significativo na magnitude de corrente utilizando-se o eletrodo modificado com quitosana. A maior corrente de pico obtida com eletrodo de pasta de carbono modificado com quitosana é devida à complexação de cátions $\mathrm{Cu}$ (II) pelos grupos amino e hidroxila da quitosana, tornando este sensor mais sensível para a determinação de íons $\mathrm{Cu}(\mathrm{II})$ na amostra.

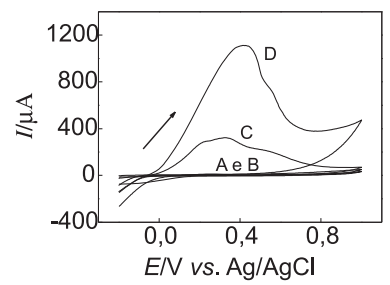

Figura 3. Voltamogramas típicos para o EPC (A e C) e o EPCM com quitosana (B e D) em solução de $\mathrm{NaNO}_{3} 0,1 \mathrm{~mol} \mathrm{~L}^{-1}$ na ausência (A e B) e na presença (C e D), respectivamente, de Cu(II) $1,0 \times 10^{-3} \mathrm{~mol} \mathrm{~L}^{-1} ; v=50 \mathrm{mV} \mathrm{s}^{-1}, a 25^{\circ} \mathrm{C}$

\section{Mecanismo proposto}

A Figura 4 apresenta o esquema proposto das reações do eletrodo modificado com quitosana para a determinação de $\mathrm{Cu}$ (II). De acordo com a literatura ${ }^{24,30-34}$ e os resultados experimentais obtidos no presente estudo, o mecanismo proposto baseia-se na quelação dos íons metálicos com a quitosana imobilizada na superfície do eletrodo (1). Em uma segunda etapa (2), os íons metá- licos complexados na superfície do eletrodo são reduzidos a cobre metálico no potencial de $-0,2 \mathrm{~V}$ vs. $\mathrm{Ag} / \mathrm{AgCl}\left(\mathrm{KCl} 3 \mathrm{~mol} \mathrm{~L}^{-1}\right) \mathrm{du}-$ rante 270 s. Finalmente, utilizando voltametria de redissolução anódica em um intervalo de potencial de $-0,2$ a $0,5 \mathrm{~V}$, a uma velocidade de varredura de potencial de $25 \mathrm{mV} \mathrm{s}^{-1}$, ocorre a oxidação de $\mathrm{Cu}(0)$ adsorvido na superfície do eletrodo a $\mathrm{Cu}(\mathrm{II})$, resultando em um sinal analítico proporcional à concentração de $\mathrm{Cu}(0)$ oxidado a $\mathrm{Cu}(\mathrm{II})$ ou proporcional à concentração de $\mathrm{Cu}$ (II) presente na solução (3).

$$
\mathrm{Cu}^{2+}{ }_{(\mathrm{aq})}+\mathrm{QTS}_{(\mathrm{sup})} \longrightarrow\left[\mathrm{Cu}^{2+}-\mathrm{QTS}_{(\text {sup })}\right.
$$

$$
\left[\mathrm{Cu}^{2+}-\mathrm{QTS}\right]_{(\text {sup })}+2 \mathrm{e}^{-} \longrightarrow\left[\mathrm{Cu}^{0}-\mathrm{QTS}\right]_{(\text {sup })}
$$

$$
\left[\mathrm{Cu}^{0}-\mathrm{QTS}_{(\text {sup })} \longrightarrow \mathrm{Cu}^{2+}{ }_{(\mathrm{aq})}+2 \mathrm{e}^{-}+\mathrm{QTS}_{(\text {sup })}\right.
$$

Figura 4. Mecanismo proposto para a resposta voltamétrica do EPCM com quitosana: (1) etapa de complexação de $\mathrm{Cu}(\mathrm{II})$ pela quitosana imobilizada no eletrodo; (2) redução de $\mathrm{Cu}(\mathrm{II})$ a $\mathrm{Cu}(0)$ na superfície do eletrodo na etapa de pré-concentração por 270 s e (3) etapa de redissolução anódica (oxidação de $\mathrm{Cu}(0)$ a $\mathrm{Cu}(\mathrm{II}))$

\section{Estudo dos interferentes em potencial}

Estudou-se a interferência em potencial de diversos cátions metálicos nas medidas de voltametria de redissolução anódica na presença $\mathrm{Cu}$ (II) $1,0 \times 10^{-3} \mathrm{~mol} \mathrm{~L}^{-1}$. Variaram-se as concentrações dos interferentes de $1,0 \times 10^{-8}$ a $1,0 \times 10^{-3} \mathrm{~mol} \mathrm{~L}^{-1}$. As concentrações dos cátions concomitantes que apresentaram uma interferência igual ou menor que 5\% estão indicadas na Tabela 1. Observa-se uma interferência significativa do mercúrio na determinação de íons $\mathrm{Cu}(\mathrm{II})$, limitando assim o emprego desse sensor para amostras possuindo concentrações de $\mathrm{Hg}$ (II) igual ou inferior a $1,0 \times 10^{-8} \mathrm{~mol} \mathrm{~L}^{-1}$.

Tabela 1. Efeito dos possíveis interferentes em potencial na determinação de $\mathrm{Cu}(\mathrm{II})$

\begin{tabular}{lc}
\hline Cátion metálico & [cátion] $\left(\mathrm{mol} \mathrm{L}^{-1}\right)$ \\
\hline Alcalinos e alcalino-terrosos & $1,0 \times 10^{-3}$ \\
$\mathrm{Cd}(\mathrm{II})$ & $1,6 \times 10^{-5}$ \\
$\mathrm{~Pb}(\mathrm{II})$ & $1,0 \times 10^{-5}$ \\
$\mathrm{Co}(\mathrm{II})$ & $1,0 \times 10^{-5}$ \\
$\mathrm{Ni}(\mathrm{II})$ & $1,5 \times 10^{-5}$ \\
$\mathrm{Cr}(\mathrm{III})$ & $1,2 \times 10^{-5}$ \\
$\mathrm{Hg}(\mathrm{II})$ & $1,0 \times 10^{-8}$ \\
\hline
\end{tabular}

${ }^{*}[\mathrm{Cu}(\mathrm{II})]=1,0 \times 10^{-6} \mathrm{~mol} \mathrm{~L}^{-1}$

\section{Curva analítica}

Após otimização das condições de trabalho para o eletrodo de pasta de carbono modificado com quitosana, realizaram-se medidas voltamétricas de redissolução anódica no intervalo de potencial de $-0,2$ a $0,5 \mathrm{~V}$ (vs $\mathrm{Ag} / \mathrm{AgCl}$ ) e intervalo de concentração de cobre de $2,0 \times 10^{-7}$ a $7,4 \times 10^{-6} \mathrm{~mol} \mathrm{~L}^{-1}$, a fim de se obter uma curva analítica. A Figura 5 apresenta os voltamogramas de redissolução anódica obtidos nas condições experimentais descritas.

A equação da curva analítica é representada por $\Delta I_{\mathrm{pa}}(\mu \mathrm{A})=9,8$ $+4,8 \times 10^{6}[\mathrm{Cu}(\mathrm{II})]\left(\mathrm{mol} \mathrm{L}^{-1}\right), \mathrm{r}=0,994$ para o intervalo de concen- 


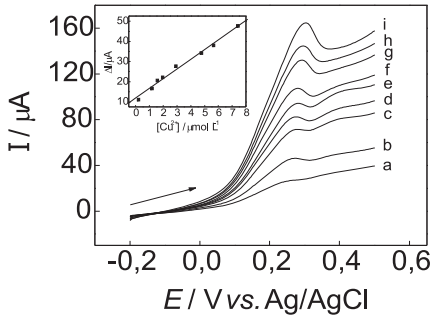

Figura 5. Voltamogramas obtidos empregando-se o EPCM com quitosana $25 \%$ $(\mathrm{m} / \mathrm{m})$ para (a) branco e soluções de Cu(II) variando de 2,0 $\times 10^{7}$ a 7,4 $\times 10^{-6} \mathrm{~mol}$ $L^{-1}$ (i) em solução de $\mathrm{NaNO}_{3} 0,1 \mathrm{~mol} \mathrm{~L}^{-1} ; \mathrm{pH}=6,5, v=25 \mathrm{mV} \mathrm{s}^{-1}$, a $25^{\circ} \mathrm{C}$

tração de 2,0 x $10^{-7}$ a 7,4 x $10^{-6} \mathrm{~mol} \mathrm{~L} \mathrm{~L}^{-1}$, com limite de detecção de $8,3 \times 10^{-8} \mathrm{~mol} \mathrm{~L}^{-1}((3 \mathrm{X} \mathrm{SD}) / \mathrm{S})$ onde SD é o desvio padrão do branco $(0,133)$ e $S$ a inclinação da curva analítica.

Foram realizados estudos de repetibilidade e reprodutibilidade utilizando as condições otimizadas já descritas anteriormente. Nos estudos de reprodutibilidade do EPCM com quitosana na presença de íons $\mathrm{Cu}(\mathrm{II})$, utilizaram-se três diferentes sensores realizando-se medidas em triplicata para uma concentração de $\mathrm{Cu}$ (II) $1,0 \times 10^{-6}$ mol L-1, obtendo-se um desvio padrão relativo (RSD) menor que 5\%. Estudou-se também a repetibilidade do EPCM com quitosana, nas mesmas condições mencionadas, realizando-se diversas medidas utilizando o mesmo eletrodo imerso na solução contendo íons $\mathrm{Cu}(\mathrm{II})$. Após o uso contínuo do eletrodo, sem a renovação da superfície, obteve-se um desvio padrão relativo menor que $5 \%(n=10)$ para solução de $\mathrm{Cu}(\mathrm{II}) 1,0 \times 10^{-6} \mathrm{~mol} \mathrm{~L}^{-1}$.

\section{Aplicação}

O método proposto foi utilizado para a determinação de íons $\mathrm{Cu}(\mathrm{II})$ em duas amostras de águas residuárias de laboratório e os resultados comparados com a técnica de espectrometria de absorção atômica (AAS). A Tabela 2 apresenta os resultados obtidos destas determinações. Cálculos estatísticos dos resultados obtidos mostraram boa concordância entre os métodos a um nível de confiança de $95 \%$, indicando que o eletrodo proposto pode ser utilizado na determinação de íons $\mathrm{Cu}(\mathrm{II})$.

Tabela 2. Análise da amostra utilizando o método proposto e o método comparativo (AAS)

\begin{tabular}{lccc}
\hline Amostra & \multicolumn{2}{c}{$\mathrm{Cu}(\mathrm{II}) / \mathrm{mol} \mathrm{L}^{-1}$} & Erro Relativo \% \\
& Comparativo & Proposto & \\
\hline A & $1,4 \times 10^{-6}$ & $1,5 \times 10^{-6}$ & $+7,1$ \\
B & $2,1 \times 10^{-6}$ & $2,2 \times 10^{-6}$ & $+4,8$ \\
\hline
\end{tabular}

\section{CONCLUSÕES}

O eletrodo de pasta de carbono modificado com quitosana pode ser empregado para a determinação de $\mathrm{Cu}$ (II) presente em águas residuárias no intervalo de $2,0 \times 10^{-7}$ a $7,4 \times 10^{-6} \mathrm{~mol} \mathrm{~L}^{-1}$, empregando-se voltametria de redissolução anódica. Além disso, o procedimento proposto é barato, simples e não necessita de nenhum tipo de tratamento da amostra podendo ser aplicado em laboratórios de análise de rotina. $\mathrm{O}$ limite de detecção encontrado é adequa- do para a análise de $\mathrm{Cu}(\mathrm{II})$ em águas residuárias atendendo às exigências estabelecidas pelas agências de controle ambiental $(\text { CONAMA })^{35}$.

\section{MATERIAL SUPLEMENTAR}

Está disponível gratuitamente em http://quimicanova.sbq.org.br, na forma de arquivo PDF.

\section{AGRADECIMENTOS}

À FAPESP, ao CNPq e à CAPES pelo suporte financeiro, bem como à CAPES pela bolsa de doutorado de L. H. Marcolino Jr e à FAPESP pela bolsa de iniciação científica de B. C. Janegitz.

\section{REFERÊNCIAS}

1. Galli, A.; de Souza, D.; Garbellini, G. S.; Coutinho, C. F. B.; Mazo, L. H.; Avaca, L. A.; Machado, S. A. S.; Quim. Nova 2006, 29, 105.

2. de Souza, D.; Codognoto, L.; Malagutti, A. R.; Toledo, R. A.; Pedrosa, V. A.; Oliveira, R. T. S.; Mazo, L. H.; Avaca, L. A.; Machado, S. A. S.; Quim. Nova 2004, 27, 790 .

3. Cox, J. A.; Tess, M. E.; Cummings, T. E.; Rev. Anal. Chem. 1996, 15, 173

4. Gilmartin, M. A. T.; Hart, J. P. ; Analyst 1995, 120, 1029.

5. Kalcher, K.; Kauffmann, J. M.; Wang, J.; Svancara, I.; Vytras, K.; Neuhold, C.; Yang, Z.; Electroanalysis 1995, 7, 5.

6. Santos, A. D.; Gorton, L.; Kubota, L. T.; Electrochim. Acta 2002 , 47, 3351

7. Wang, J.; Electroanalysis 1991, 3, 255.

8. Katz, E.; Lotzbeyer, T.; Schlereth, D. D.; Schuhmann, W.; Schmidt, H. L.; J. Electroanal. Chem. 1994, 373, 189.

9. Rosatto, S. S.; Freire, R. S.; Duran, N.; Kubota, L. T.; Quim. Nova 2001, 24, 77.

10. Adams, R. N.; Anal. Chem. 1958, 30, 1576.

11. Ravichandran, K.; Baldwin, R. P.; J. Electroanal. Chem. 1981, 126, 293.

12. Pereira, A. C.; Santos, A. D.; Kubota, L. T.; Quim. Nova 2002, 25, 1012.

13. Babel, S.; Kurniawan, T. A.; J. Hazard. Mater. 2003, 97, 219.

14. Laus, R.; Laranjeira, M. C. M.; Martins, A. O.; Favere, V. T.; Pedrosa, R. C.; Benassi, J. C.; Geremias, R.; Quim. Nova 2006, 29, 34.

15. Janegitz, B. C.; Lourenção, B. C.; Lupetti, K. O.; Fatibello-Filho, O.; Quim. Nova 2007, 30, 879.

16. Kumar, M.; React. Funct. Polym. 2000, 46, 1.

17. Kurita, K.; Mar. Biotechnol. 2006, 8, 203.

18. Liu, N.; Song, J. F.; Anal. Bioanal. Chem. 2005, 383, 358.

19. Varma, A. J.; Deshpande, S. V.; Kennedy, J. F.; Carbohydr. Polym. 2004, 55, 77.

20. Abdullah, J.; Ahmad, M.; Karuppiah, N.; Heng, L. Y.; Sidek, H.; Sens. Actuators, B 2006, 114, 604 .

21. Magalhaes, J.; Machado, A.; Talanta 1998, 47, 183.

22. Qian, L.; Yang, X. R.; Talanta 2006, 68, 721

23. Zhang, M.; Mullens, C.; Gorski, W.; Electrochim. Acta 2005, 51, 4528.

24. de Oliveira, I.; Vieira, I. C.; Quim. Nova 2006, 29, 932.

25. de Oliveira, I.; Vieira, I. C.; Lupetti, K. O.; Fatibello-Filho, O.; de Favere, V. T.; Laranjeira, M. C. M.; Anal. Lett. 2004, 37, 3111.

26. Tan, X. C.; Tian, Y. X.; Cai, P. X.; Zou, X. Y.; Anal. Bioanal. Chem. 2005, 381,500 .

27. de Oliveira, I.; Fernandes, S. C.; Vieira, I. C.; J. Pharm. Biomed. Anal. 2006, $41,366$.

28. Lima, I. S.; Lazarin, A. M.; Airoldi, C.; Int. J. Biol. Macromol. 2005, 36, 79.

29. Lu, H. Q.; He, X. W.; Zeng, X. S.; Wan, Q. J.; Zhang, Z. Z.; Talanta 2003, $59,553$.

30. Ben-Shalom, N.; Kudabaeva, N.; Borisover, M.; Chemosphere 2005, 59, 1309.

31. de Sousa, E. R.; Marques, E. P.; Fernandes, E. N.; Zhang, J. J.; Marques, A. L. B.; J. Braz. Chem. Soc. 2006, 17, 177.

32. Lima, I. S.; Lazarin, A. M.; Airoldi, C.; Carbohydr. Polym. 2006, 64, 385.

33. Vieira, R. S.; Beppu, M. M.; Water Res. 2006, 40, 1726.

34. Ye, X. Z.; Yang, Q. H.; Wang, Y.; Li, N. Q.; Talanta 1998, 47, 1099.

35. http://www.mma.gov.br/port/conama/index.cfm, acessada em Outubro 2006 


\section{DETERMINAÇÃo VOLTAMÉTRICA POR REDISSOLUÇÃO ANÓDICA DE Cu(II) EM ÁGUAS RESIDUÁRIAS} EMPREGANDO UM ELETRODO DE PASTA DE CARBONO MODIFICADO COM QUITOSANA

Bruno Campos Janegitz , Luiz Humberto Marcolino Junior e Orlando Fatibello-Filho*

Departamento de Química, Universidade Federal de São Carlos, CP 676, 13560-970 São Carlos - SP, Brasil

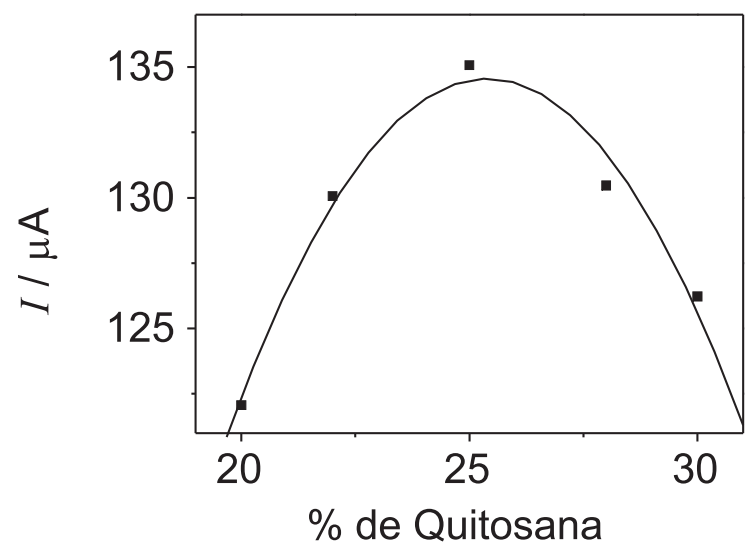

Figura 1S. Estudo da variação da concentração do biopolímero no eletrodo de pasta de carbono modificado com quitosana, em meio de $\mathrm{NaNO}_{3} 0,1 \mathrm{~mol}$ $L^{-1} ; v=25 \mathrm{mV} \mathrm{s}^{-1}$, a $25^{\circ} \mathrm{C}$, na presença de $\mathrm{Cu}(\mathrm{II}) 1,0 \times 10^{-4} \mathrm{~mol} \mathrm{~L}^{-1}$

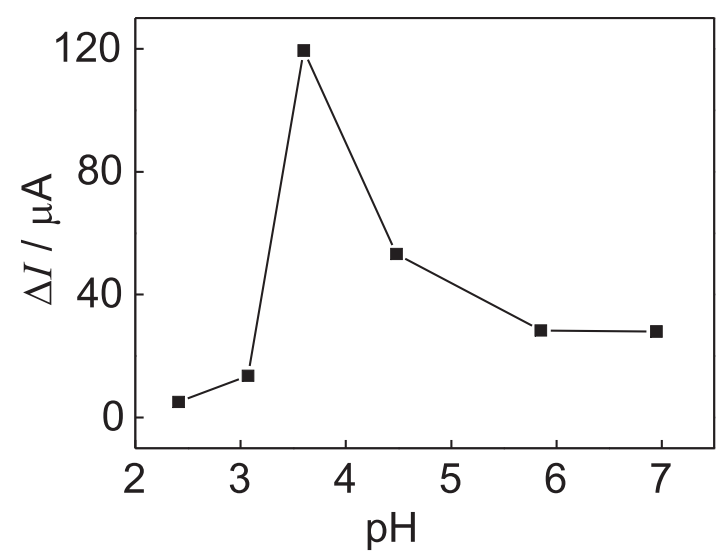

Figura 2S. Estudo da variação do pH em meio de $\mathrm{NaNO}_{3} 0,1 \mathrm{~mol} \mathrm{~L}^{-1} ; v=25$ $\mathrm{mV} \mathrm{s} \mathrm{s}^{-1}$, a $25^{\circ} \mathrm{C}$, na presença de $\mathrm{Cu}$ (II) $1,0 \times 10^{-4} \mathrm{~mol} \mathrm{~L}^{-1}$

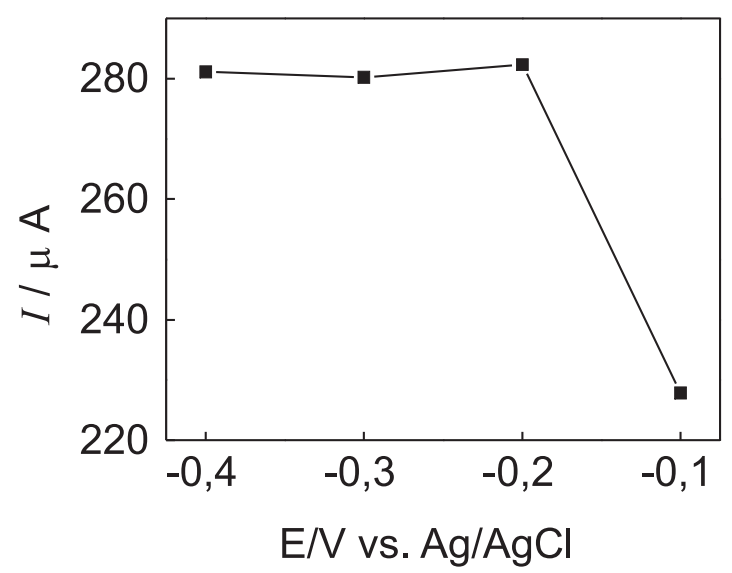

Figura 3S. Estudo do potencial de pré-acúmulo no intervalo de $-0,1$ a $-0,3 \mathrm{~V}$, em meio de de $\mathrm{NaNO}_{3} 0,1 \mathrm{~mol} \mathrm{~L}^{-1} ; \mathrm{pH}=6,5, v=25 \mathrm{mV} \mathrm{s}^{-1}$, a $25^{\circ} \mathrm{C}$, na presença de $\mathrm{Cu}$ (II) $1,0 \times 10^{-4} \mathrm{~mol} \mathrm{~L}^{-1}$

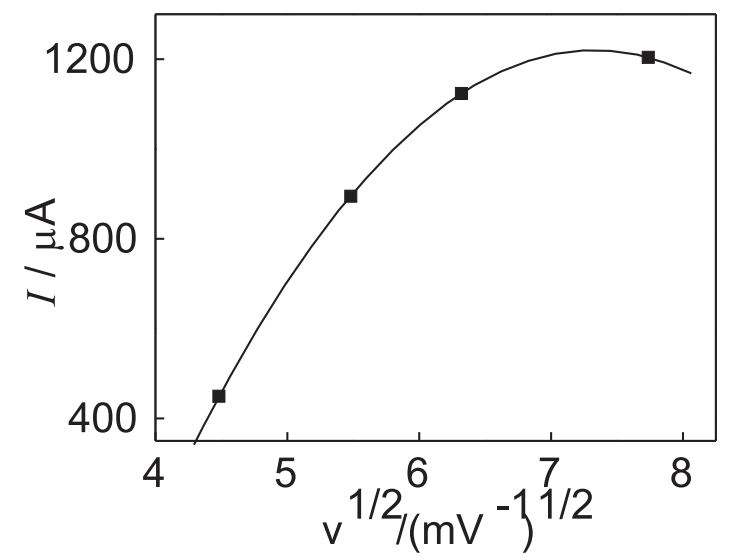

Figura 4S. Efeito da velocidade de varredura no processo adsortivo das espécies na superfície do eletrodo referente ao processo de oxidação do analito, na presença de $\mathrm{Cu}^{2+} 1,8 \times 10^{-5} \mathrm{em} \mathrm{NaNO}_{3} \mathrm{O}, 1 \mathrm{~mol} \mathrm{~L}^{-1}(\mathrm{pH}=6,5)$ 